\title{
Anticollocated Backstepping Observer Design for a Class of Coupled Reaction-Diffusion PDEs
}

\author{
Antonello Baccoli and Alessandro Pisano \\ Department of Electrical and Electronic Engineering, University of Cagliari, 09123 Cagliari, Italy \\ Correspondence should be addressed to Antonello Baccoli; antonello.baccoli@diee.unica.it
}

Received 10 September 2015; Accepted 4 November 2015

Academic Editor: Ai-Guo Wu

Copyright (C) 2015 A. Baccoli and A. Pisano. This is an open access article distributed under the Creative Commons Attribution License, which permits unrestricted use, distribution, and reproduction in any medium, provided the original work is properly cited.

The state observation problem is tackled for a system of $n$ coupled reaction-diffusion PDEs, possessing the same diffusivity parameter and equipped with boundary sensing devices. Particularly, a backstepping-based observer is designed and the exponential stability of the error system is proven with an arbitrarily fast convergence rate. The transformation kernel matrix is derived in the explicit form by using the method of successive approximations, thereby yielding the observer gains in the explicit form, too. Simulation results support the effectiveness of the suggested design.

\section{Introduction}

Model-based control and advanced process monitoring of Distributed-Parameter Systems (DPSs), governed by Partial Differential Equations (PDEs), typically require full state information. However, the available measurements of DPS are typically located on the boundary of the spatial domain that motivates the need of the state observer $[1,2]$.

For linear infinite-dimensional systems the Luenberger observer theory was established by replacing matrices with linear operators [2-4], and the observer design was confined to determining a gain operator that stabilizes the associated error dynamics. In contrast to finite dimensional systems, finding such a gain operator is not trivial even numerically because operators were not generally represented with a finite number of parameters.

Design methods, which are not relying on any discretization or finite-dimensional approximation (thereby preserving the infinite-dimensional representation of the system during the entire design process) and which are yielding the observer gains in the explicit form, have only recently been investigated. In this context, the backstepping method appears to be a particularly effective systematic design approach which can be applied for a broad class of systems governed by PDEs $[5,6]$. Basically, the backstepping approach relies on the application of an invertible Volterra integral transformation mapping, a predefined exponentially stable target system, into the observer error dynamics.

For systems governed by parabolic PDEs defined on a one-dimensional (1D) spatial domain, a systematic observer design approach using boundary sensing is introduced in [6] . Recently, the backstepping-based observer design was presented in [7] for reaction-diffusion processes with spatially varying reaction coefficient and a certain weighted average of the state over the spatial domain as measured output. In $[8,9]$, backstepping-based observer design was addressed for reaction-diffusion processes evolving in multidimensional spatial domains. In [10], the backstepping-based design for parabolic processes was applied by adopting a nonconventional target system for the error dynamics, embedding certain discontinuous output injection terms.

More recently, high-dimensional systems of coupled PDEs were considered in the backstepping-based boundary control and observer design settings. The most intensive efforts of the current literature seem however to be oriented towards coupled hyperbolic processes of the transport type [11-15]. In [14], a $2 \times 2$ linear hyperbolic system was stabilized by a single observer-based boundary control input, with an additional feature that an unmatched disturbance, generated by an a priori known exosystem, was rejected. Both 
the controller and the observer were designed by following the backstepping approach. In [12] a state estimator in a semiinfinite three-dimensional (3D) domain is presented for a coupled model of magnetohydrodynamic flow, and Fourier transform methods were applied to put the system in a form, where the $1 \mathrm{D}$ backstepping method is applicable. In [15], a backstepping-based observer was designed for a system of two diffusion-convection-reaction processes coupled through the corresponding boundary conditions. In [13], a $2 \times 2$ system of coupled linear heterodirectional hyperbolic systems was stabilized by a backstepping-based observercontroller under some boundedness restriction on the spatially dependent coupling coefficients. In [11], observer-controller design was studied for a system of $n+1$ coupled firstorder heterodirectional hyperbolic linear PDEs ( $n$ of which featured rightward convecting transport and one leftward) with a single boundary input. Some specific results concerning the backstepping-based output feedback boundary stabilization of parabolic coupled PDEs have been presented in the literature. In [16] the controller/observer design for the linearized $2 \times 2$ model of thermal-fluid convection has been treated.

In this work, the observer design is developed for a class of $n$ coupled diffusion-reaction PDEs in the 1D spatial domain $x \in[0,1]$. The task of the present paper is to generalize some results presented in [6], where explicit backstepping observers were developed for a scalar unstable reactiondiffusion equation. Here a generalization is made for a set of $n$ reaction-diffusion processes, which are coupled through the corresponding reaction terms. The motivation to this investigation comes from chemical processes [17] where coupled temperature-concentration parabolic PDEs were involved to describe system dynamics. This generalization is shown to be far from being trivial because the underlying backsteppingbased treatment gives rise to more complex development of finding out an explicit form of the observer gains in the form of matrix Bessel series, and, furthermore, it turns out to be unfeasible in the general case where each process possesses its own diffusivity parameter. In this work we therefore address the simplified case where all processes possess the same diffusivity value, and we postpone the more general case for further investigations (see Remark 2). The present paper can be considered as the observer design counterpart of our recent work [18], where the stabilizing boundary controller design problem was addressed for a similar class of systems differing only in the boundary conditions from that considered in the present work. Subsequently, in [19], the stabilizing boundary control design problem in the general case of different diffusivity parameters was addressed and solved.

Particularly, in the present context, two output injections are needed in the observer dynamics (one distributed along the spatial domain and another one located at the uncontrolled boundary).

The structure of the paper is as follows. After introducing some useful notation in Section 1.1, Section 2 states the problem to be investigated and introduces the proposed observer structure with the underlying backstepping transformation and (matrix) kernel PDE. In Section 3, the explicit solution of the kernel PDE is derived. In Section 4, the proposed observer design is summarized and the main result of this paper is presented. Section 5 discusses supporting simulation results, and Section 6 collects some concluding remarks and future perspectives of this research.

1.1. Notation. The notation used throughout is fairly standard. $L_{2}(0,1)$ stands for the Hilbert space of square integrable scalar functions $z(\zeta)$ on $(0,1)$ with the corresponding norm

$$
\|z(\cdot)\|_{2}=\sqrt{\int_{0}^{1} z^{2}(\zeta) d \zeta .}
$$

Throughout the paper the notation

$$
\left[L_{2}(0,1)\right]^{n}=\underbrace{L_{2}(0,1) \times L_{2}(0,1) \times \cdots \times L_{2}(0,1)}_{n \text { times }}
$$

is also utilized and

$$
\|Z(\cdot)\|_{2, n}=\sqrt{\sum_{i=1}^{n}\left\|z_{i}(\cdot)\right\|_{2}^{2}}
$$

stands for the corresponding norm of a generic vector function $Z(\zeta)=\left[z_{1}(\zeta), z_{2}(\zeta), \ldots, z_{n}(\zeta)\right] \in\left[L_{2}(0,1)\right]^{n}$.

With reference to a generic real-valued symmetric matrix $W$ of dimension $n, \sigma_{1}(W)$ denotes the smallest eigenvalue of $W$. Finally, $I_{n \times n}$ stands for the identity matrix of dimension $n$.

\section{Problem Formulation and Backstepping Transformation}

The following $n$-dimensional system of coupled reactiondiffusion processes, equipped with Neumann-type boundary conditions and governed by the boundary-value problem

$$
\begin{aligned}
& Q_{t}(x, t)=\theta Q_{x x}(x, t)+\Lambda Q(x, t), \\
& Q_{x}(0, t)=0 \\
& Q(1, t)=U(t),
\end{aligned}
$$

is under study. Hereinafter,

$$
\begin{aligned}
Q(x, t) & =\left[q_{1}(x, t), q_{2}(x, t), \ldots, q_{n}(x, t)\right]^{T} \\
& \in\left[L_{2}(0,1)\right]^{n}
\end{aligned}
$$

is the vector collecting the state of all systems,

$$
U(t)=\left[u_{1}(t), u_{2}(t), \ldots, u_{n}(t)\right]^{T} \in \Re^{n}
$$

is the boundary input vector, $\Lambda=\left\{\lambda_{i j}\right\} \in \mathfrak{R}^{n \times n}$ is a realvalued square matrix, and $\theta \in \mathfrak{R}^{+}$is a positive scalar. The open-loop system $(4)-(6)$ (with $U(t)=0$ ) may possess arbitrarily many unstable eigenvalues when the symmetric part $\left(\Lambda+\Lambda^{T}\right) / 2$ of matrix $\Lambda$ possesses sufficiently large 
positive eigenvalues. For system (4)-(6) of $n$ coupled reaction-diffusion processes, the following observer

$$
\begin{aligned}
& \widehat{Q}_{t}(x, t)= \theta \widehat{Q}_{x x}(x, t)+\Lambda \widehat{Q}(x, t) \\
&+G(x)[Q(0, t)-\widehat{Q}(0, t)], \\
& \widehat{Q}_{x}(0, t)= M[Q(0, t)-\widehat{Q}(0, t)], \\
& \widehat{Q}(1, t)=U(t)
\end{aligned}
$$

is proposed with $G(x)$ being a $n$th order square matrix of observer gain functions and $M \in \mathfrak{R}^{n, n}$ being a square matrix of constant observer gains. The error variable

$$
\widetilde{Q}(x, t)=Q(x, t)-\widehat{Q}(x, t)
$$

is then governed by the error system

$$
\begin{aligned}
& \widetilde{Q}_{t}(x, t)=\theta \widetilde{Q}_{x x}(x, t)+\Lambda \widetilde{Q}(x, t)-G(x) \widetilde{Q}(0, t), \\
& \widetilde{Q}_{x}(0, t)=-M \widetilde{Q}(0, t), \\
& \widetilde{Q}(1, t)=0 .
\end{aligned}
$$

To design the observer gains $G(x)$ and $M$, the backstepping approach is involved to find out an invertible transformation

$$
\widetilde{Q}(x, t)=\widetilde{Z}(x, t)-\int_{0}^{x} P(x, y) \widetilde{Z}(y, t) d y,
$$

where $P(x, y)$ is a $n \times n$ matrix kernel function whose elements are denoted as $p_{i j}(x, y), i, j=1,2, \ldots, n$, which maps the error system (11)-(13) into the exponentially stable (the exponential stability properties of the target error system (15)-(17) will be investigated in Theorem 4) target error dynamics

$$
\begin{aligned}
& \widetilde{Z}_{t}(x, t)=\theta \widetilde{Z}_{x x}(x, t)-\bar{C} \widetilde{Z}(x, t), \\
& \widetilde{Z}_{x}(0, t)=0, \\
& \widetilde{Z}(1, t)=0 .
\end{aligned}
$$

The following lemma is in order.

Lemma 1. The error system (11)-(13) is transferred by (14) into the target error dynamics (15)-(17) provided that the design terms $M$ and $G(x)$ are selected as

$$
\begin{gathered}
G(x)=\theta P_{y}(x, 0), \\
M=P(0,0),
\end{gathered}
$$

where $P(x, y)$ is a solution to the kernel PDE

$$
\begin{aligned}
& P_{x x}(x, y)-P_{y y}(x, y) \\
& \quad=-\frac{1}{\theta}[P(x, y) \bar{C}+\Lambda P(x, y)], \\
& P(x, x)=\frac{\Lambda+\bar{C}}{2 \theta}(x-1), \\
& P(1, y)=0 .
\end{aligned}
$$

Proof. Employing the Leibnitz differentiation rule, the spatial differentiation of (14) results in

$$
\begin{aligned}
\widetilde{Q}_{x}(x, t)= & \widetilde{Z}_{x}(x, t)-P(x, x) \widetilde{Z}(x, t) \\
& -\int_{0}^{x} P_{x}(x, y) \widetilde{Z}(y, t) d y, \\
\widetilde{Q}_{x x}(x, t)= & \widetilde{Z}_{x x}(x, t)-\left[\frac{d}{d x} P(x, x)\right] \widetilde{Z}(x, t) \\
& -P(x, x) \widetilde{Z}_{x}(x, t)-P_{x}(x, x) \widetilde{Z}(x, t) \\
& -\int_{0}^{x} P_{x x}(x, y) \widetilde{Z}(y, t) d y .
\end{aligned}
$$

In turn, the temporal differentiation of (14) and recursive integration by parts yield

$$
\begin{aligned}
\widetilde{Q}_{t}(x, t)= & \widetilde{Z}_{t}(x, t)-\int_{0}^{x} P(x, y) \widetilde{Z}_{t}(y, t) d y \\
= & \widetilde{Z}_{t}(x, t)-P(x, x) \theta \widetilde{Z}_{x}(x, t) \\
& +\theta P(x, 0) \widetilde{Z}_{x}(0, t)+\theta P_{y}(x, x) \widetilde{Z}(x, t) \\
& -\theta P_{y}(x, 0) \widetilde{Z}(0, t) \\
& -\theta \int_{0}^{x} P_{y y}(x, y) \widetilde{Z}(y, t) d y \\
& +\int_{0}^{x} P(x, y) \bar{C} \widetilde{Z}(y, t) d y .
\end{aligned}
$$

By evaluating (14) at $x=0$ and $x=1$ and considering (17), one derives that

$$
\begin{aligned}
& \widetilde{Q}(0, t)=\widetilde{Z}(0, t), \\
& \widetilde{Q}(1, t)=-\int_{0}^{1} P(1, y) \widetilde{Z}(y, t) d y .
\end{aligned}
$$

Substituting (14), (16) and (24)-(26) into (11) and performing lengthy but straightforward computations yield

$$
\begin{aligned}
& \widetilde{Z}_{t}(x, t)-\theta \widetilde{Z}_{x x}(x, t)+\bar{C} \widetilde{Z}(x, t) \\
& =-\left\{\theta\left[\frac{d}{d x} P(x, x)\right]+\theta P_{y}(x, x)+\theta P_{x}(x, x)\right. \\
& -\Lambda-\bar{C}\} \widetilde{Z}(x, t)+[P(x, x) \theta-\theta P(x, x)] \widetilde{Z}_{x}(x, t) \\
& +\left[\theta P_{y}(x, 0)-G(x)\right] \widetilde{Z}(0, t)+\int_{0}^{x}\left[\theta P_{y y}(x, y)\right. \\
& \left.-\theta P_{x x}(x, y)-P(x, y) \bar{C}-\Lambda P(x, y)\right] \\
& \cdot \widetilde{Z}(y, t) d y .
\end{aligned}
$$

By evaluating (23) at $x=0$ and considering (16), it follows that

$$
\widetilde{Q}_{x}(0, t)=-P(0,0) \widetilde{Z}(0, t) .
$$


Substituting (29) and (26)-(27) into (12) and (13), one derives the conditions

$$
\begin{aligned}
& {[M-P(0,0)] \widetilde{Z}(0, t)=0,} \\
& \int_{0}^{1} P(1, y) \widetilde{Z}(y, t) d y=0 .
\end{aligned}
$$

Clearly, to obtain the target error PDE (15) the right hand side of (28) should be identically zero. To meet this requirement, it suffices to employ relations (30) and exploit the identity $(d / d x) P(x, x)=P_{x}(x, x)+P_{y}(x, x)$, thereby obtaining both the kernel boundary-value problem

$$
\begin{aligned}
& \theta\left(P_{x x}(x, y)-P_{y y}(x, y)\right) \\
& \quad=-P(x, y) \bar{C}-\Lambda P(x, y), \\
& 2 \theta \frac{d}{d x} P(x, x)=\Lambda+\bar{C} \\
& P(1, y)=0
\end{aligned}
$$

and the observer gain design conditions in the form of (18)(19). Integrating (32) with respect to $x$ and considering (19) result in

$$
\begin{aligned}
P(x, x) & =\frac{1}{2 \theta}(\Lambda+\bar{C}) x+P(0,0) \\
& =\frac{1}{2 \theta}(\Lambda+\bar{C}) x+M
\end{aligned}
$$

Evaluating (34) at $x=1$ yields

$$
P(1,1)=\frac{1}{2 \theta}(\Lambda+\bar{C})+M
$$

By evaluating (33) at $y=1$ it is concluded that $P(1,1)=0$, thus getting from (35) that

$$
M=-\frac{1}{2 \theta}(\Lambda+\bar{C}) .
$$

Considering (34) and (36), one finally rewrites (31)-(33) in the form of (20)-(22). Lemma 1 is proven.

Remark 2. The present paper is confined to the case in which all the coupled PDEs (4) possess the same diffusivity parameter $\theta$. The reason behind this is that in the more general case where each process has its own diffusivity $\theta_{i}$ $(i=1,2, \ldots, n)$, the corresponding "generalized" version

$$
\begin{aligned}
& \Theta\left(P_{x x}(x, y)-P_{y y}(x, y)\right) \\
& \quad=-P(x, y) \bar{C}-\Lambda P(x, y), \\
& \Theta \frac{d}{d x} P(x, x)+\Theta P_{x}(x, x)+\Theta P_{y}(x, x)=\Lambda+\bar{C}, \\
& P(x, x) \Theta=\Theta P(x, x), \\
& P(1, y)=0
\end{aligned}
$$

of (20)-(22), where $\Theta=\operatorname{diag}\left(\theta_{i}\right)$, sets an overdetermined boundary-value problem that has no solution, unless specific constraints are imposed on the matrix $\bar{C}$ and on the form of the kernel matrix $P(x, y)$. This topic calls for further investigation and will be published elsewhere.

\section{Solving the Kernel PDE (20)-(22)}

For later use, the following result is reproduced.

Theorem 3. Problem (20)-(22) possesses a solution

$$
\begin{aligned}
P(x, y)= & -\sum_{n=0}^{\infty} \frac{2(1-x)\left((1-y)^{2}-(1-x)^{2}\right)^{n}}{n !(n+1) !} \\
& \cdot\left(\frac{1}{4 \theta}\right)^{n+1}\left[\sum_{i=0}^{n}\left(\begin{array}{l}
n \\
i
\end{array}\right) \Lambda^{i}(\Lambda+\bar{C}) \bar{C}^{n-i}\right]
\end{aligned}
$$

which is of class $C^{\infty}$ in the domain $0 \leq y \leq x \leq 1$.

Proof. By the invertible change of variables

$$
\begin{aligned}
& \bar{x}=1-y, \\
& \bar{y}=1-x,
\end{aligned}
$$

one transforms (20)-(22) into

$$
\begin{aligned}
& \bar{P}_{\overline{x x}}(\bar{x}, \bar{y})-\bar{P}_{\overline{y y}}(\bar{x}, \bar{y}) \\
& \quad=\frac{1}{\theta}[\bar{P}(\bar{x}, \bar{y}) \bar{C}+\Lambda \bar{P}(\bar{x}, \bar{y})], \\
& \bar{P}(\bar{x}, \bar{x})=-\frac{\Lambda+\bar{C}}{2 \theta} \bar{x}, \\
& \bar{P}(\bar{x}, 0)=0 .
\end{aligned}
$$

Following [20], the existence of a solution to problem (40) can be shown by transforming it into an integral equation using the change of the variables

$$
\begin{gathered}
\xi=x+y, \\
\eta=x-y .
\end{gathered}
$$

Setting

$$
H(\xi, \eta)=\bar{P}(x, y)=\bar{P}\left(\frac{\xi+\eta}{2}, \frac{\xi-\eta}{2}\right)
$$

the relations

$$
\begin{aligned}
\bar{P}_{x} & =H_{\xi}+H_{\eta}, \\
\bar{P}_{x x} & =H_{\xi \xi}+2 H_{\xi \eta}+H_{\eta \eta}, \\
\bar{P}_{y} & =H_{\xi}-H_{\eta}, \\
\bar{P}_{y y} & =H_{\xi \xi}-2 H_{\xi \eta}+H_{\eta \eta}
\end{aligned}
$$


are obtained, and the matrix kernel boundary-value problem (40), written in the new coordinates, takes the form

$$
\begin{aligned}
H_{\xi \eta}(\xi, \eta) & =\frac{1}{4 \theta} H(\xi, \eta) \bar{C}+\frac{1}{4 \theta} \Lambda H(\xi, \eta), \\
H(\xi, 0) & =-\frac{1}{4 \theta}(\Lambda+\bar{C}) \xi \\
H(\xi, \xi) & =0 .
\end{aligned}
$$

Integrating (44) with respect to $\eta$ from 0 to $\eta$ and considering the relation $H_{\xi}(\xi, 0)=-(1 / 4 \theta)(\Lambda+\bar{C})$, which follows from (45), one obtains

$$
\begin{aligned}
H_{\xi}(\xi, \eta)= & -\frac{1}{4 \theta}(\Lambda+\bar{C}) \\
& +\frac{1}{4 \theta} \int_{0}^{\eta}[H(\xi, s) \bar{C}+\Lambda H(\xi, s)] d s .
\end{aligned}
$$

Integrating (47) with respect to $\xi$ from $\eta$ to $\xi$ yields

$$
\begin{aligned}
& \int_{\eta}^{\xi} H_{\tau}(\tau, \eta) d \tau \\
& =\int_{\eta}^{\xi}-\frac{1}{4 \theta}(\Lambda+\bar{C}) d \tau \\
& \quad+\frac{1}{4 \theta} \int_{\eta}^{\xi}\left\{\int_{0}^{\eta}[H(\tau, s) \bar{C}+\Lambda H(\tau, s)] d s\right\} d \tau
\end{aligned}
$$

which can further be manipulated to

$$
\begin{aligned}
H(\xi, \eta)-H(\eta, \eta) \\
=-\frac{1}{4 \theta}(\Lambda+\bar{C})(\xi-\eta) \\
\quad+\frac{1}{4 \theta} \int_{\eta}^{\xi}\left\{\int_{0}^{\eta}[H(\tau, s) \bar{C}+\Lambda H(\tau, s)] d s\right\} d \tau .
\end{aligned}
$$

An explicit form of $H(\eta, \eta)$ is subsequently derived. For this purpose, (46) is used to obtain

$$
H(\eta, \eta)=0
$$

By substituting (50) into (49) one derives an integral equation for $H(\xi, \eta)$ :

$$
\begin{aligned}
& H(\xi, \eta) \\
& =-\frac{1}{4 \theta}(\Lambda+\bar{C})(\xi-\eta) \\
& \quad+\frac{1}{4 \theta} \int_{\eta}^{\xi}\left\{\int_{0}^{\eta}[H(\tau, s) \bar{C}+\Lambda H(\tau, s)] d s\right\} d \tau .
\end{aligned}
$$

The method of successive approximations is then applied to show that (51) has a smooth solution. Let us start with an initial approximation

$$
H^{0}(\xi, \eta)=0
$$

and set up the recursive formula for (51) as follows:

$$
\begin{aligned}
H^{n+1}(\xi, \eta) & \\
= & -\frac{1}{4 \theta}(\Lambda+\bar{C})(\xi-\eta) \\
& +\frac{1}{4 \theta} \int_{\eta}^{\xi}\left\{\int_{0}^{\eta}\left[H^{n}(\tau, s) \bar{C}+\Lambda H^{n}(\tau, s)\right] d s\right\} d \tau .
\end{aligned}
$$

Provided that this recursion converges, solution $H(\xi, \eta)$ can be represented as

$$
H(\xi, \eta)=\lim _{n \rightarrow \infty} H^{n}(\xi, \eta)
$$

Let

$$
\Delta H^{n}(\xi, \eta)=H^{n+1}(\xi, \eta)-H^{n}(\xi, \eta)
$$

stand for the difference between two consecutive terms. Then, the recursion

$$
\begin{aligned}
& \Delta H^{0}(\xi, \eta)=H^{1}(\xi, \eta)=-\frac{1}{4 \theta}(\Lambda+\bar{C})(\xi-\eta), \\
& \Delta H^{n+1}(\xi, \eta) \\
& \quad=\frac{1}{4 \theta} \int_{\eta}^{\xi}\left\{\int_{0}^{\eta}\left[\Delta H^{n}(\tau, s) \bar{C}+\Lambda \Delta H^{n}(\tau, s)\right] d s\right\} d \tau
\end{aligned}
$$

is correspondingly concluded from (52)-(53) and (54) is alternatively represented as

$$
H(\xi, \eta)=\sum_{n=0}^{\infty} \Delta H^{n}(\xi, \eta) .
$$

Since variables $\xi$ and $\eta$ lie in the bounded domain $0 \leq \eta \leq$ $\xi \leq 2$, one can apply (56) to show that

$$
\left\|\Delta H^{0}(\xi, \eta)\right\| \leq \frac{1}{\theta}(\|\Lambda\|+\|\bar{C}\|)=N .
$$

In order to apply the mathematical induction method, suppose that

$$
\left\|\Delta H^{n}(\xi, \eta)\right\| \leq N^{n+1} \frac{(\xi+\eta)^{n}}{n !} .
$$

Then, by employing (57), (59), and (60) one arrives at

$$
\begin{aligned}
& \left\|\Delta H^{n+1}(\xi, \eta)\right\| \leq \frac{1}{4 \theta}(\|\Lambda\|+\|\bar{C}\|) \\
& \cdot \frac{N^{n+1}}{n !}\left|2 \int_{0}^{\eta} \int_{0}^{\tau}(\tau+s)^{n} d s d \tau+\int_{\eta}^{\xi} \int_{0}^{\eta}(\tau+s)^{n} d s d \tau\right| \\
& =\frac{N^{n+2}}{4 n !} \mid 2 \int_{0}^{\eta} \int_{0}^{\tau}(\tau+s)^{n} d s d \tau \\
& +\int_{\eta}^{\xi} \int_{0}^{\eta}(\tau+s)^{n} d s d \tau \mid .
\end{aligned}
$$


It is readily shown (cf. [21], equation (2.14)) that the next estimate

$$
\begin{aligned}
& \left|2 \int_{0}^{\eta} \int_{0}^{\tau}(\tau+s)^{n} d s d \tau+\int_{\eta}^{\xi} \int_{0}^{\eta}(\tau+s)^{n} d s d \tau\right| \\
& \quad \leq 4 \frac{(\xi+\eta)^{n+1}}{(n+1)}
\end{aligned}
$$

holds. Therefore, combining (61) and (62), one gets

$$
\left\|\Delta H^{n+1}(\xi, \eta)\right\| \leq N^{n+2} \frac{(\xi+\eta)^{n+1}}{(n+1) !} .
$$

Thus, by mathematical induction, (63) holds for all $n \geq 0$. It then follows from the Weierstrass $M$-test that the series (58) converges absolutely and uniformly in $0 \leq \eta \leq \xi \leq 2$. By (56)(57), it follows that

$$
\begin{aligned}
& \Delta H^{1}(\xi, \eta) \\
& \quad=-\frac{\xi^{2} \eta+\xi \eta^{2}}{2}\left(\frac{1}{4 \theta}\right)^{2}[(\Lambda+\bar{C}) \Lambda+\bar{C}(\Lambda+\bar{C})] .
\end{aligned}
$$

Iterating on the computations, one observes the pattern which leads to the following formula:

$$
\begin{aligned}
\Delta H^{n}(\xi, \eta)= & -\frac{(\xi \eta)^{n}(\xi-\eta)}{n !(n+1) !}\left(\frac{1}{4 \theta}\right)^{n+1} \\
& \cdot\left[\sum_{i=0}^{n}\left(\begin{array}{l}
n \\
i
\end{array}\right) \Lambda^{i}(\Lambda+\bar{C}) \bar{C}^{n-i}\right] .
\end{aligned}
$$

The solution to the integral equation (51) is therefore given by the next series expansion

$$
\begin{aligned}
H(\xi, \eta)= & -\sum_{n=0}^{\infty} \frac{(\xi \eta)^{n}(\xi-\eta)}{n !(n+1) !}\left(\frac{1}{4 \theta}\right)^{n+1} \\
& \cdot\left[\sum_{i=0}^{n}\left(\begin{array}{l}
n \\
i
\end{array}\right) \Lambda^{i}(\Lambda+\bar{C}) \bar{C}^{n-i}\right]
\end{aligned}
$$

which is absolutely and uniformly converging.

Converting (66) into the original $x, y$ variables, one obtains the series expansion (38) for the Kernel matrix $P(x, y)$ which solves the kernel boundary-value problem (40). Straightforward inspection reveals that (38) is infinitely times continuously differentiable. Returning back to the original $(x, y)$ variables, one obtains (38). Theorem 3 is thus proven.

3.1. Inverse Transformation. Transformation (14) is a matrix Volterra integral equation of the second type. Since $P(x, y)$ is continuous by Theorem 3 , there exists a continuous inverse kernel $L(x, y)$ (see, e.g., $[11,22]$ for the scalar case which is straightforwardly extended to the present vector case) such that

$$
\widetilde{Q}(x, t)=\widetilde{Z}(x, t)+\int_{0}^{x} L(x, y) \widetilde{Z}(y, t) d y
$$

implicitly defined on $T=\left\{(x, y) \in R^{2}: 0 \leq y \leq x \leq 1\right\}$ by

$$
L(x, y)=P(x, y)+\int_{y}^{x} L(x, s) P(s, y) d s .
$$

Relation (68) can in fact be easily derived by substituting (14) into (67) and performing straightforward manipulations of the resulting integral equation. The method of successive approximations can be then applied to show that (68) gives rise to a unique $R(x, y)$, which has as much regularity as $P(x, y)$ has. Detailed computations, which follow similar steps as those carried out in the proof of Theorem 3, are skipped for brevity.

\section{Main Result}

Taking advantage of the explicit solution (38) to the kernel boundary-value problem (20)-(22), the explicit representation

$$
\begin{aligned}
& M=-\frac{\Lambda+\bar{C}}{2 \theta} \\
& G(x)=\theta \sum_{n=0}^{\infty} \frac{4 n(1-x)\left(2 x-x^{2}\right)^{n-1}}{n !(n+1) !}\left(\frac{1}{4 \theta}\right)^{n+1} \\
& \cdot\left[\sum_{i=0}^{n}\left(\begin{array}{l}
n \\
i
\end{array}\right) \Lambda^{i}(\Lambda+\bar{C}) \bar{C}^{n-i}\right]
\end{aligned}
$$

of the observer gains is straightforwardly derived by specifying (18)-(19) accordingly.

The stability features of the target error dynamics (15)(17) are going to be studied. The following result is in force.

Theorem 4. If the design matrix $\bar{C}$ is selected such that its symmetric part $\bar{C}_{s}=\left(\bar{C}+\bar{C}^{T}\right) / 2$ is positive definite, then system (15)-(17) is exponentially stable in the space $\left[L_{2}(0,1)\right]^{n}$ with the convergence rate specified by

$$
\|\widetilde{Z}(\cdot, t)\|_{2, n} \leq\|\widetilde{Z}(\cdot, 0)\|_{2, n} e^{-\sigma_{1}\left(\bar{C}_{s}\right) t} .
$$

Proof. Consider the Lyapunov function $V(t)=(1 / 2) \int_{0}^{1} \widetilde{Z}^{T}(\xi$, t) $\widetilde{Z}(\xi, t) d \xi=(1 / 2)\|\widetilde{Z}(\cdot, t)\|_{2, n}^{2}$. The corresponding time derivative along the solutions of (15)-(17) is given by

$$
\begin{aligned}
\dot{V}(t)= & \int_{0}^{1} \widetilde{Z}^{T}(\xi, t) \Theta \widetilde{Z}_{x x}(\xi, t) d \xi \\
& -\int_{0}^{1} \widetilde{Z}^{T}(\xi, t) \bar{C} \widetilde{Z}(\xi, t) d \xi .
\end{aligned}
$$

Integrating by parts taking into account the BCs (16) and (17) and exploiting the diagonal form of matrix $\Theta$ yield

$$
\begin{aligned}
\int_{0}^{1} \widetilde{Z}^{T}(\xi, t) \Theta \widetilde{Z}_{x x}(\xi, t) d \xi \\
=\left.\widetilde{Z}^{T}(\chi, t) \Theta \widetilde{Z}_{x}(\chi, t)\right|_{\chi=0} ^{\chi=1} \\
\quad-\int_{0}^{1} \widetilde{Z}_{x}^{T}(\xi, t) \Theta \widetilde{Z}_{x}(\xi, t) d \xi \leq-\theta_{m}\left\|\widetilde{Z}_{x}(\cdot, t)\right\|_{2, n}^{2},
\end{aligned}
$$


where $\theta_{m}=\min _{1 \leq i \leq n} \theta_{i}>0$. Since $\sigma_{1}\left(\bar{C}_{s}\right)$ is assumed to be positive then exploiting the trivial inequality $\widetilde{Z}^{T}(\xi, t) \bar{C} \widetilde{Z}(\xi$, $t) \geq \sigma_{1}\left(\bar{C}_{s}\right)^{T} \widetilde{Z}(\xi, t) \widetilde{Z}(\xi, t)$ and employing (72), one manipulates (71) to derive

$$
\begin{aligned}
\dot{V}(t) & \leq-\theta_{m}\left\|\widetilde{Z}_{\xi}(\cdot, t)\right\|_{2, n}^{2}-2 \sigma_{1}\left(\bar{C}_{s}\right) V(t) \\
& \leq-2 \sigma_{1}\left(\bar{C}_{s}\right) V(t),
\end{aligned}
$$

thereby concluding the exponential stability of the target error dynamics in the space $\left[L_{2}(0,1)\right]^{n}$ with a convergence rate obeying the estimate (70). Theorem 4 is proven.

The next theorem specifies the proposed observer design and summarizes the main result of this paper.

Theorem 5. The observer (9), with gains $M$ and $G(x)$ set as in (69) and with matrix $\bar{C}$ being selected such that its symmetric part $\bar{C}_{s}=\left(\bar{C}+\bar{C}^{T}\right) / 2$ is positive definite, reconstructs the state of system (4)-(6) with an arbitrarily fast convergence rate in accordance with

$$
\|\widetilde{Q}(\cdot, t)\|_{2, n} \leq A\|\widetilde{Q}(\cdot, 0)\|_{2, n} e^{-\sigma_{1}\left(C_{s}\right) t},
$$

where $A$ is a positive constant independent of $\widetilde{Q}(\xi, 0)$.

Proof. In Lemma 1 and Theorem 3, it was shown that the error system (11)-(13) is transferred, by means of (14), into the target error dynamics (15)-(17) provided that the gains $M$ and $G(x)$ are selected as in (18)-(19) where solution $P(x, y)$ to kernel PDE (20)-(22) is given by (38). Specifying (18)-(19) in light of the actual form of solution (38), it straightforwardly results in (69), where $P(0,0)$ is derived by specifying $(21)$ at $x=0$ and $P_{y}(x, 0)$ is readily obtained by differentiating (38) with respect to $y$ at $y=0$.

The asymptotic stability features of (15)-(17), subject to the design requirement that the arbitrary design parameter $\bar{C}_{s}=\left(\bar{C}+\bar{C}^{T}\right) / 2$ is positive definite, were demonstrated in Theorem 4. In particular, according to (70), the corresponding convergence rate can be made arbitrarily fast by a proper selection of the $\bar{C}$ matrix.

From now on, we follow [20] to derive analogous convergence properties for the original system (4)-(6) as well. Observing that $\xi+\eta=x$, one derives from (58)-(60) that $\|P(x, y)\| \leq N e^{2 N x}$, and the same bound can be derived for the norm of the inverse transformation kernel matrix $L(x, y)$ as well; that is, $\|L(x, y)\| \leq N e^{2 N x}$. A straightforward generalization of [20, Th 4] yields that those two boundedness relations, coupled together, establish the equivalence of norms of $\widetilde{Z}(x, t)$ and $\widetilde{Q}(x, t)$ in $\left[L_{2}(0,1)\right]^{n}$ which means that there exists a positive constant $A$ independent of $\widetilde{Q}(\xi, 0)$ such that the estimate (74) is in force as a direct consequence of (70). Theorem 5 is proven.

\section{Simulation Results}

5.1. Academic Example. To validate the proposed observer, system (4)-(6) of coupled reaction-diffusion processes is specified for simulation purposes with $n=3$ and with parameters

$$
\begin{aligned}
\theta & =2, \\
\Lambda & =\left[\begin{array}{lll}
1 & 2 & 3 \\
4 & 5 & 3 \\
2 & 5 & 1
\end{array}\right] .
\end{aligned}
$$

The initial conditions are set to $q_{1}(x, 0)=q_{2}(x, 0)=$ $q_{3}(x, 0)=2 \sin (\pi x)+2 \sin (3 \pi x)$. For solving the underlying PDEs, a standard finite-difference approximation method is used by discretizing the spatial solution domain $x \in[0,1]$ into a finite number of $N$ uniformly spaced solution nodes $x_{i}=i h, h=1 /(N+1), i=1,2, \ldots, N$. The value $N=40$ is then used. The resulting 40th order discretized system is subsequently solved by fixed-step Runge-Kutta ODE4 method with step $T_{s}=10^{-4}$.

The unstable behaviour of the plant subject to the openloop input vector $U(t)=[5 \sin t, 10 \sin 2 t, 15 \sin 3 t]^{T}$ is displayed in Figure 1, which for certainty shows the diverging spatiotemporal evolution of the states $q_{1}(x, t)$ and $q_{3}(x, t)$.

Observer (9), (69) has been implemented by selecting the design matrix $\bar{C}=10 I_{3 \times 3}$ and by specifying the initial conditions at $\widehat{q}_{1}(x, 0)=\widehat{q}_{2}(x, 0)=\widehat{q}_{3}(x, 0)=0$. Figure 2 displays the spatiotemporal evolution of the observed states $\widehat{q}_{1}(x, t)$ and $\widehat{q}_{3}(x, t)$, which clearly mimic the corresponding actual states. Figure 3 shows the temporal evolution of the norm $\|\widetilde{Q}(\cdot, t)\|_{2,3}$, which tends to zero exponentially, thus confirming the correct functioning of the proposed observer and supporting the theoretical analysis.

5.2. Application Example. To provide a more valuable validation of the proposed scheme, we consider the coupled temperature-concentration dynamics of a Chemical Tubular Reactor (CTR) at low fluid superficial velocities, when convection terms become negligible, dealt with in [17]. After a suitable transformation, the next dimensionless model was derived

$$
\begin{aligned}
\frac{\partial x_{1}}{\partial t} & =D_{1} \frac{\partial^{2} x_{1}}{\partial \xi^{2}}+k_{0} \delta\left(1-x_{2}\right) e^{-\gamma /\left(1+x_{1}\right)}, \\
\frac{\partial x_{2}}{\partial t} & =D_{2} \frac{\partial^{2} x_{2}}{\partial \xi^{2}}+k_{0}\left(1-x_{2}\right) e^{-\gamma /\left(1+x_{1}\right)}, \\
x_{1 \xi}(0, t) & =x_{2 \xi}(0, t)=0, \\
x_{1}(1, t) & =u_{1}(t), \\
x_{2}(1, t) & =u_{2}(t),
\end{aligned}
$$

where the states $x_{1}$ and $x_{2}$ denote the normalized temperature and concentration, respectively, and the underlying physical parameters take the values

$$
\begin{aligned}
D_{1} & =D_{2}=0.167, \\
\delta & =0.5, \\
k_{0} & =2.426 \cdot 10^{7}, \\
\gamma & =20 .
\end{aligned}
$$




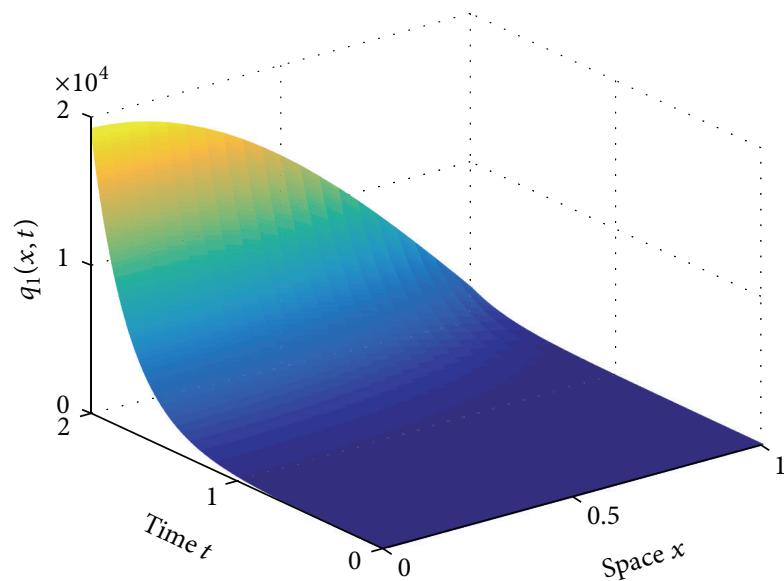

(a)

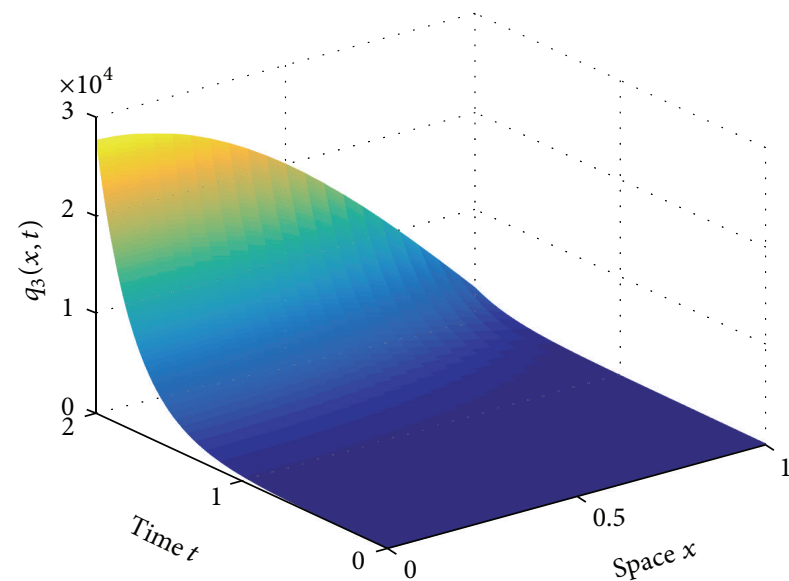

(b)

FIGURE 1: Spatiotemporal evolution of $q_{1}(x, t)$ (a) and $q_{3}(x, t)$ (b).

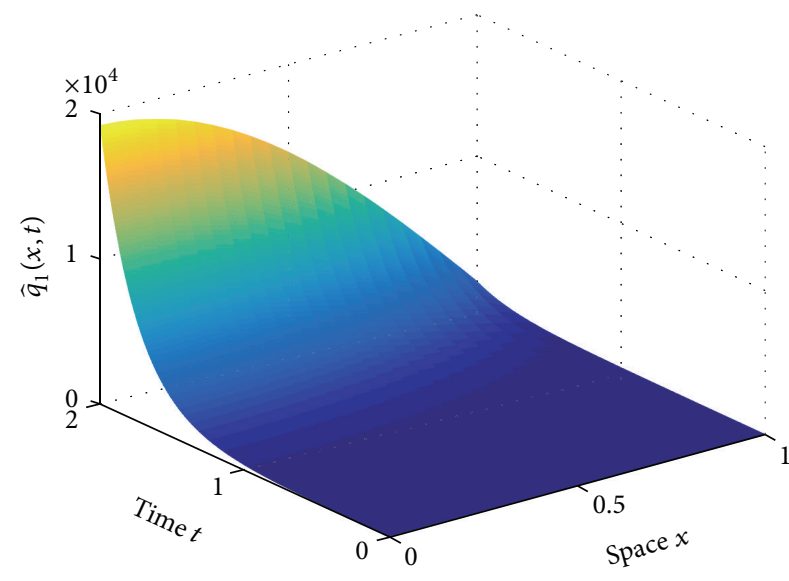

(a)

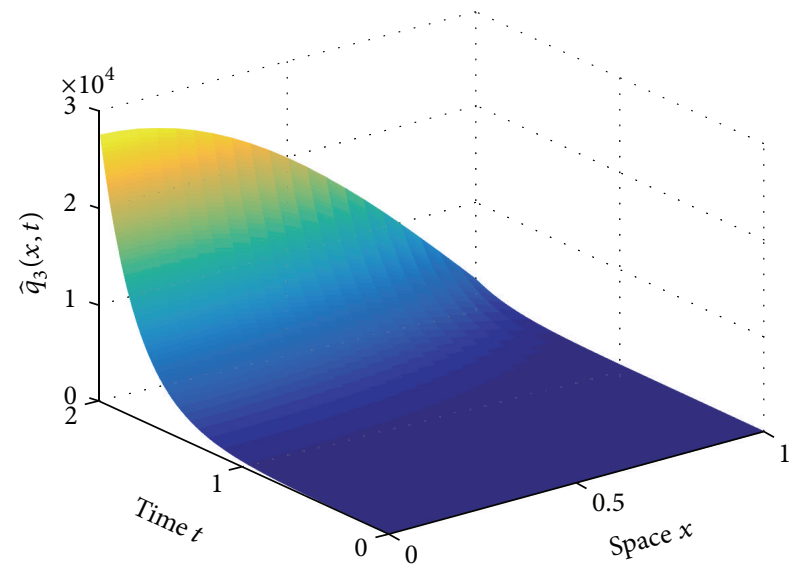

(b)

FIGURE 2: Spatiotemporal evolution of $\widehat{q}_{1}(x, t)$ (a) and $\widehat{q}_{3}(x, t)(\mathrm{b})$.

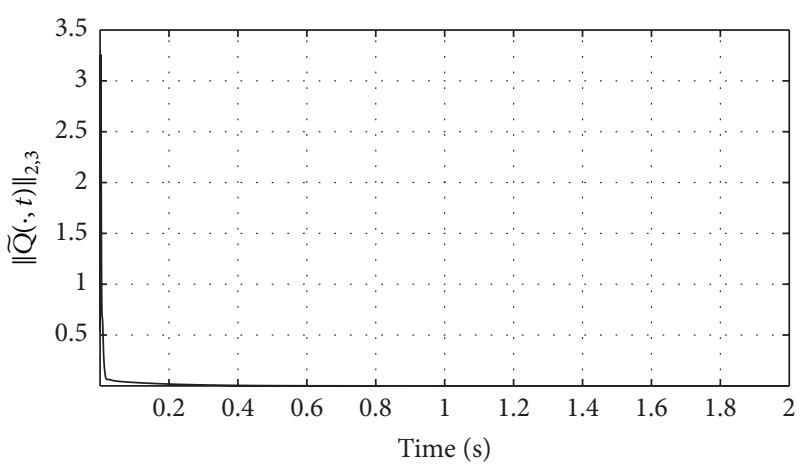

FIgURE 3: Temporal evolution of the norm $\|\widetilde{Q}(\cdot, t)\|_{2,3}$.

Its linearization around the constant profiles

$$
\begin{aligned}
& x_{1}^{*}(\xi, t)=0.1, \\
& x_{2}^{*}(\xi, t)=0.98
\end{aligned}
$$

gives rise to model (4)-(6) with the following diffusivity and reaction parameters:

$$
\begin{aligned}
\theta & =0.167, \\
\Lambda & =\left[\begin{array}{ll}
1.018 & 0.154 \\
2.037 & 0.308
\end{array}\right] .
\end{aligned}
$$

The open-loop control input $U(t)=[5 \sin t, 10 \sin 2 t]^{T}$ was selected. The plant ICs are set to $x_{1}(x, 0)=x_{2}(x, 0)=$ $2 \sin (\pi \xi)+2 \sin (3 \pi \xi)$. The unstable open-loop behaviour of the plant state $x_{2}(\xi, t)$ is displayed in Figure 4(a). Observer (9), (69) has been implemented by selecting the design matrix $\bar{C}=20 I_{2 \times 2}$ and by specifying ICs $\widehat{x}_{1}(\xi, 0)=$ $\widehat{x}_{2}(\xi, 0)=0$. Figure $4(\mathrm{~b})$ shows that the observer is able to correctly reconstruct the unstable profile of the plant state $x_{2}(\xi, t)$. Figure 5 shows the temporal evolution of the norm $\|\widetilde{Q}(\cdot, t)\|_{2,2}$, which confirms the correct functioning of the observer for the estimation of the state variable $x_{2}(\xi, t)$, too. 


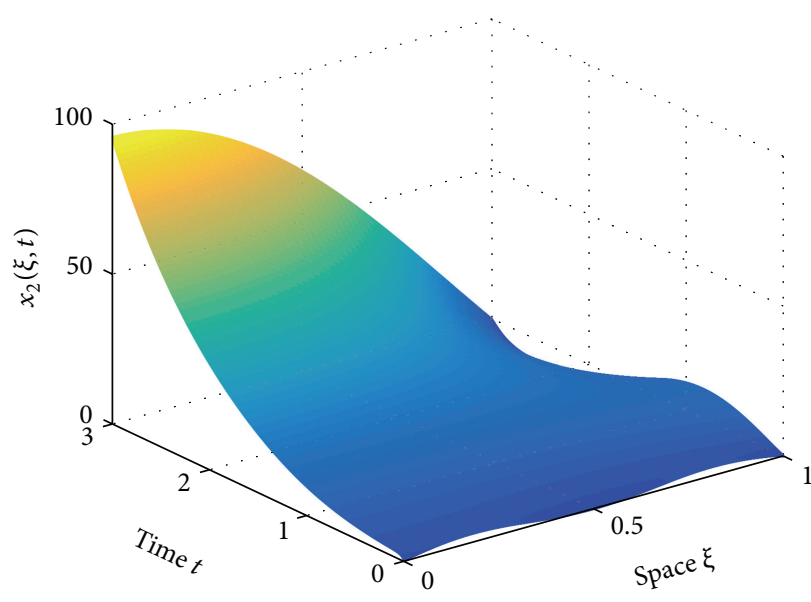

(a)

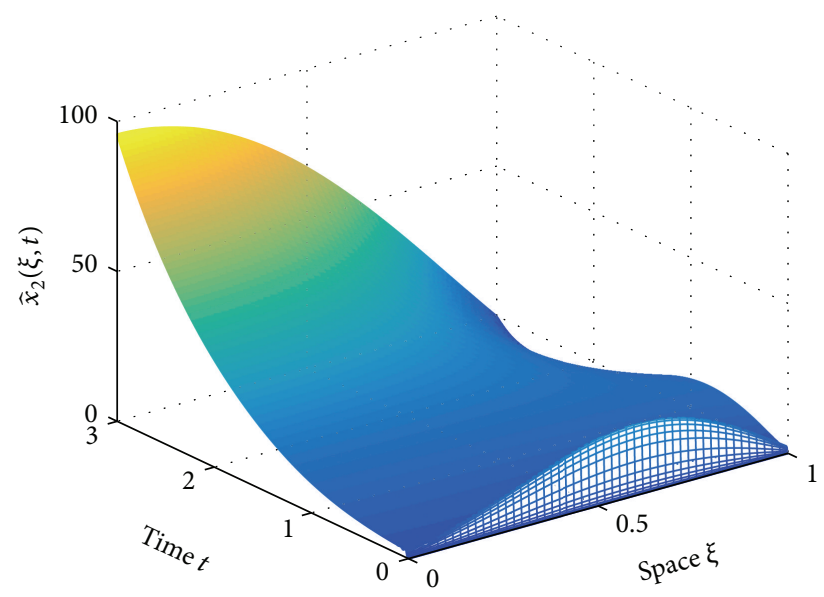

(b)

Figure 4: Spatiotemporal evolution of $x_{2}(\xi, t)$ (a) and $\widehat{x}_{2}(\xi, t)(\mathrm{b})$.

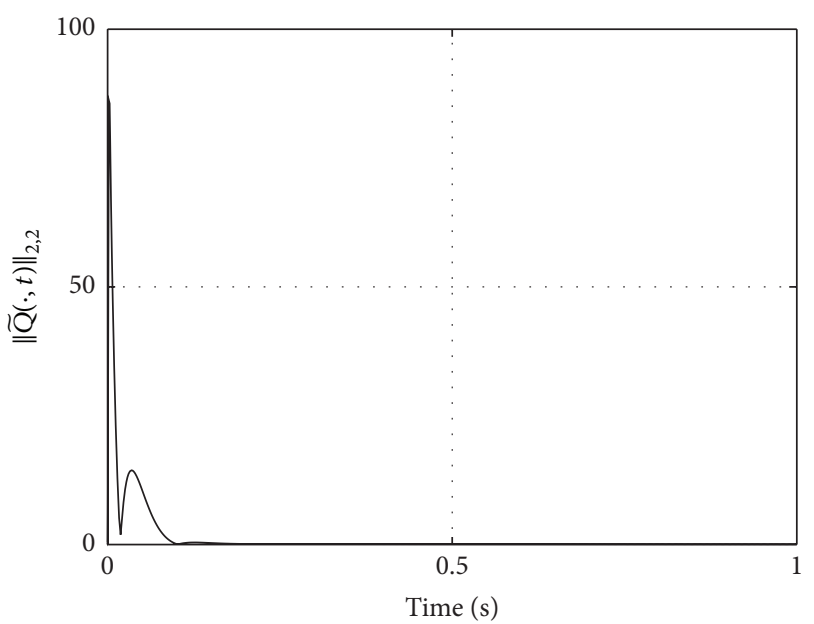

FIGURE 5: Temporal evolution of the norm $\|\widetilde{Q}(\cdot, t)\|_{2,2}$.

\section{Conclusions}

The backstepping-based anticollocated observer design of a system of $n$ coupled parabolic linear PDEs has been tackled, and an explicit representation of the underlying observer gains has been derived which allows one to enforce an arbitrarily fast exponential decay of the observation error dynamics in the space $\left[L_{2}(0,1)\right]^{n}$. The extension to the case of different diffusivities and spatially dependent parameters and the observer-based output feedback design of a stabilizing controller $U(t)$ are among the most interesting future lines of related investigations that will be pursued in our future work.

\section{Conflict of Interests}

The authors declare that there is no conflict of interests regarding the publication of this paper.

\section{Acknowledgments}

The research leading to these results has received funding from the Research Project "Modeling, Control and Experimentation of Innovative Thermal Storage Systems," funded by Sardinia Regional Government under Grant Agreement no. CRP-60193, and from the Research Project "RODEORObust Decentralised Estimation fOr Large-Scale Systems," funded by the Italian Ministry for Foreign Affairs under Grant Agreement PGR00152.

\section{References}

[1] A. Vande Wouwer and M. Zeitz, "State estimation in distributed parameter systems," in Encyclopedia of Life Support Systems (EOLSS), H. Unbehauen, Ed., Control Systems, Robotics and Automation, EOLSS Publishers, Oxford, UK, 2001.

[2] Z. Hidayat, R. Babuska, B. De Schutter, and A. Nunnez, "Observers for linear distributed-parameter systems: a survey," in Proceedings of the IEEE International Symposium on Robotic and Sensors Environments (ROSE '11), pp. 166-171, IEEE, Montreal , Canada, September 2011.

[3] R. F. Curtain and H. Zwart, An Introduction to InfiniteDimensional Linear Systems Theory, vol. 21 of Texts in Applied Mathematics, Springer, New York, NY, USA, 1995.

[4] T. Lasiecka and R. Triggiani, Control Theory for Partial Differential Equations: Continuous and Approximation Theories, I Abstract Parabolic Systems, Cambridge University Press, Cambridge, UK, 2000.

[5] M. Krstic and A. Smyshlyaev, Boundary Control of PDEs: A Course on Backstepping Designs, SIAM Advances in Design and Control, SIAM, Philadelphia, Pa, USA, 2008.

[6] A. Smyshlyaev and M. Krstic, "Backstepping observers for a class of parabolic PDEs," Systems \& Control Letters, vol. 54, no. 7, pp. 613-625, 2005.

[7] D. Tsubakino and S. Hara, "Backstepping observer design for parabolic PDEs with measurement of weighted spatial averages," Automatica, vol. 53, pp. 179-187, 2015.

[8] L. Jadachowski, T. Meurer, and A. Kugi, "State estimation for parabolic PDEs with varying parameters on 3-dimensional 
spatial domains," in Proceedings of the 18th IFAC World Congress, pp. 13338-13343, Milano, Italy, September 2011.

[9] L. Jadachowski, T. Meurer, and A. Kugi, "Backstepping observers for linear PDEs on higher-dimensional spatial domains," Automatica, vol. 51, pp. 85-97, 2015.

[10] R. Miranda, I. Chairez, and J. Moreno, "Observer design for a class of parabolic PDE via sliding modes and backstepping," in Proceedings of the 11th International Workshop on Variable Structure Systems (VSS '10), pp. 215-220, Mexico City, Mexico, June 2010.

[11] F. Di Meglio, R. Vazquez, and M. Krstic, "Stabilization of a system of coupled first-order hyperbolic linear pdes with a single boundary input," IEEE Transactions on Automatic Control, vol. 58, no. 12, pp. 3097-3111, 2013.

[12] R. Vazquez, E. Schuster, and M. Krstic, "Magnetohydrodynamic state estimation with boundary sensors," Automatica, vol. 44, no. 10, pp. 2517-2527, 2008.

[13] R. Vazquez, M. Krstic, and J.-M. Coron, "Backstepping boundary stabilization and state estimation of a $2 \times 2$ linear hyperbolic system," in Proceedings of the 50th IEEE Conference Decision and Control and European Control Conference (CDC-ECC '11), pp. 4937-4942, Orlando, Fla, USA, December 2011.

[14] O. M. Aamo, "Disturbance rejection in $2 \times 2$ linear hyperbolic systems," IEEE Transactions on Automatic Control, vol. 58, no. 5, pp. 1095-1106, 2013.

[15] S. Moura, J. Bendtsen, and V. Ruiz, "Observer design for boundary coupled PDEs: application to thermostatically controlled loads in smart grids," in Proceedings of the 52nd IEEE Conference on Decision and Control (CDC '13), pp. 6286-6291, Firenze, Italy, December 2013.

[16] R. Vazquez and M. Krstic, "Boundary observer for outputfeedback stabilization of thermal-fluid convection loop," IEEE Transactions on Control Systems Technology, vol. 18, no. 4, pp. 789-797, 2010.

[17] Y. Orlov and D. Dochain, "Discontinuous feedback stabilization of minimum-phase semilinear infinite-dimensional systems with application to chemical tubular reactor," IEEE Transactions on Automatic Control, vol. 47, no. 8, pp. 1293-1304, 2002.

[18] A. Baccoli, Y. Orlov, and A. Pisano, "On the boundary control of coupled reaction-diffusion equations having the same diffusivity parameters," in Proceedings of the IEEE 53rd Annual Conference on Decision and Control (CDC '14), pp. 5222-5228, Los Angeles, Calif, USA, December 2014.

[19] A. Baccoli, A. Pisano, and Y. Orlov, "Boundary control of coupled reaction-diffusion processes with constant parameters," Automatica, vol. 54, pp. 80-90, 2015.

[20] A. Smyshlyaev and M. Krstic, "Closed-form boundary state feedbacks for a class of 1-D partial integro-differential equations," IEEE Transactions on Automatic Control, vol. 49, no. 12, pp. 2185-2202, 2004.

[21] B.-Z. Guo and J.-J. Liu, "Sliding mode control and active disturbance rejection control to the stabilization of one-dimensional Schrödinger equation subject to boundary control matched disturbance," International Journal of Robust and Nonlinear Control, vol. 24, no. 16, pp. 2194-2212, 2014.

[22] R. Vazquez, Boundary control laws and observer design for convective, turbulent and magnetohydrodynamic flows [Ph.D. thesis], University of California, San Diego, Calif, USA, 2006. 

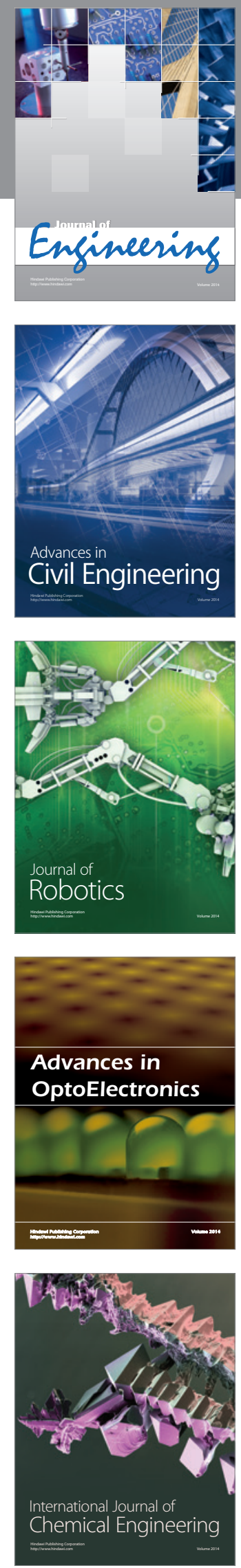

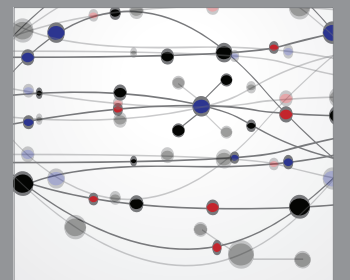

The Scientific World Journal
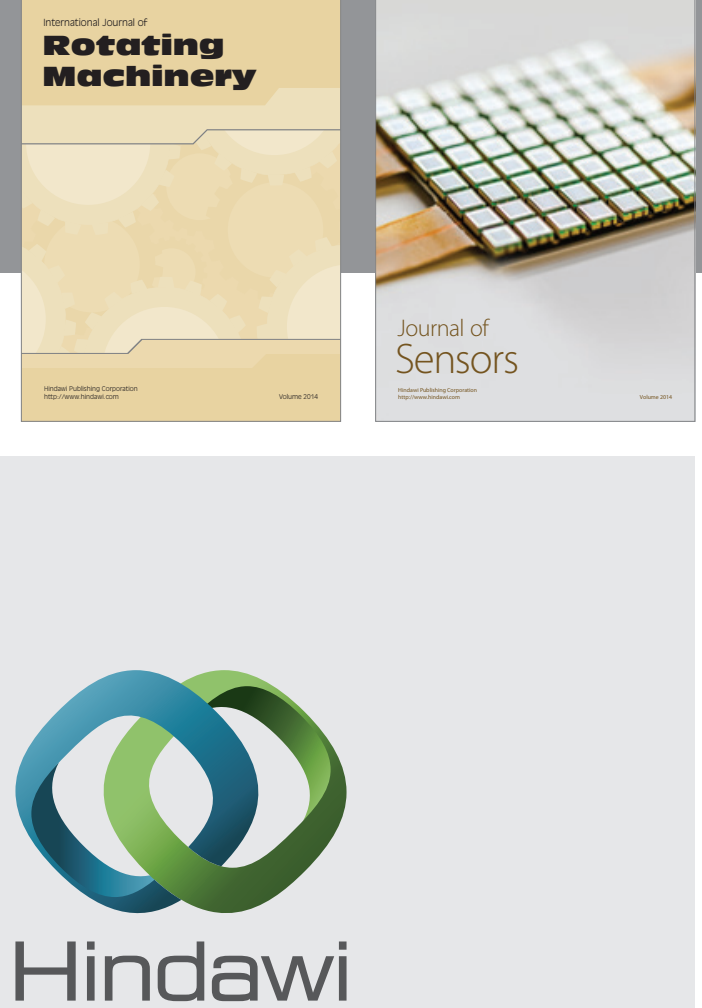

Submit your manuscripts at http://www.hindawi.com
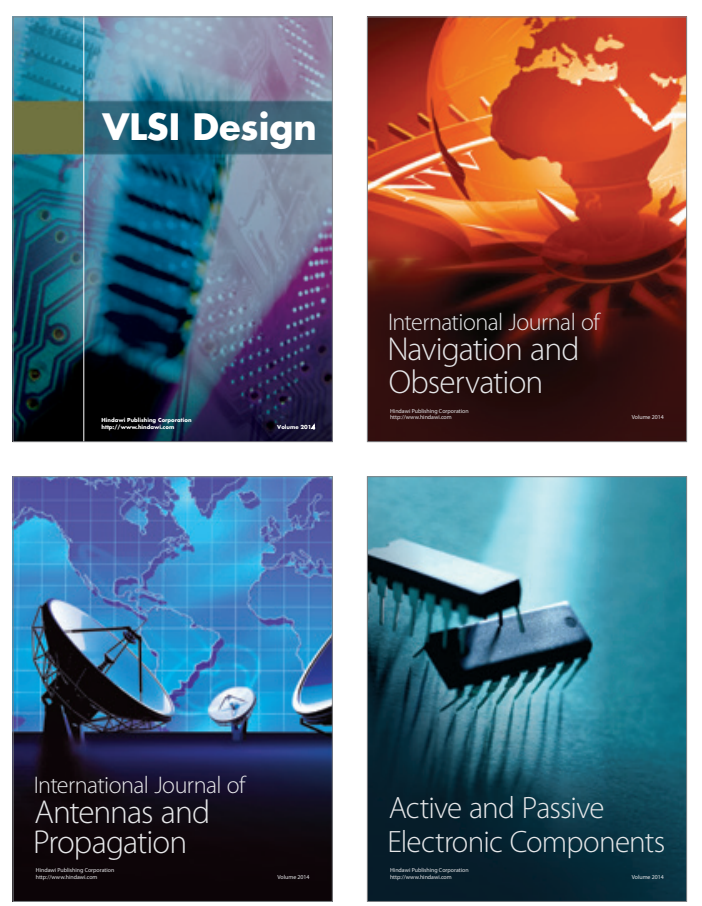
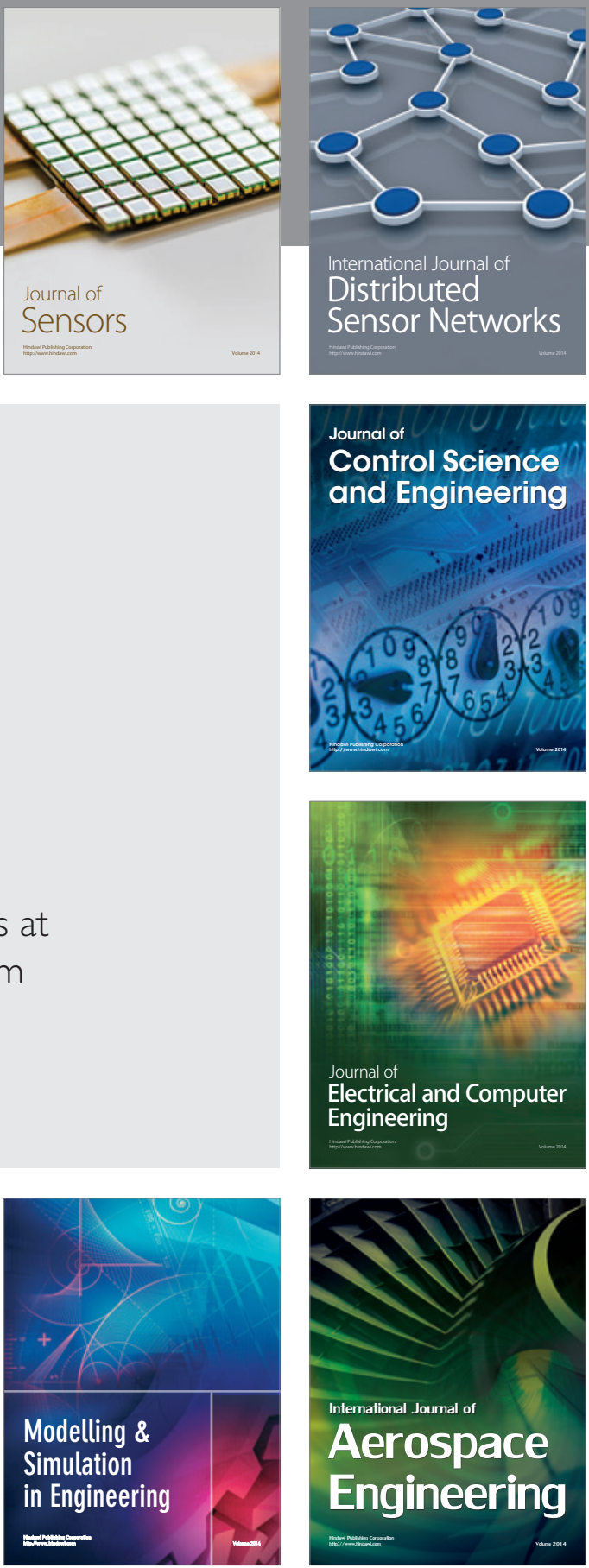

Journal of

Control Science

and Engineering
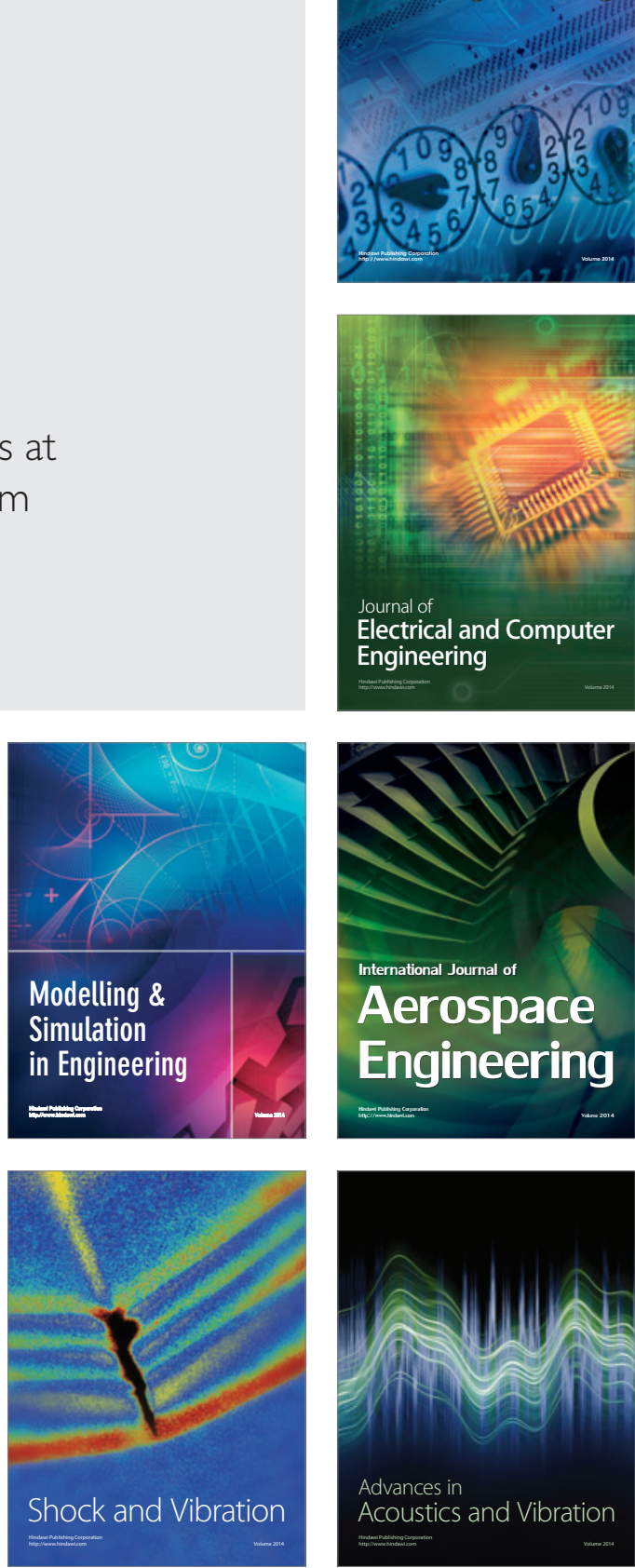